\title{
Cataract, Glaucoma, and Dry Eye Disease in Adults with Atopic Dermatitis: A Nationwide Cross-Sectional Study from the Republic of Korea
}

\author{
Sul Hee Lee, Sang-Hoon Lee, Si Hyung Lee ${ }^{1}$, Young Lip Park \\ Departments of Dermatology and ${ }^{1}$ Ophthalmology, Soonchunhyang University Bucheon Hospital, Bucheon, Korea
}

Background: Atopic dermatitis (AD) is an inflammatory skin disorder characterized by relapsing eczema with intractable itching. Ocular diseases in patients with $A D$, including cataract, retinal detachment, blepharitis, glaucoma, keratoconjunctivitis, and keratoconus, have frequently been reported worldwide. However, only a few studies using a large-sample, population-based study design have been reported so far. Objective: We investigated the association between cataract, glaucoma, and dry eye disease and AD in an adult population in the Republic of Korea. Methods: A total of 14,900 adults who participated in the Korean National Health and Nutrition Examination Survey, a nationwide, population-based, cross-sectional survey, between 2010 and 2012 were included in the study. Multiple logistic regression analyses identified the possible association between cataract, glaucoma, and dry eye disease and AD relative to matched controls. Results: After we adjusted for confounding factors in patients with AD, cataract and glaucoma were significantly associated with AD. Moreover, patients

Received April 24, 2018, Revised August 2, 2018, Accepted for publication August 14, 2018

Corresponding author: Young Lip Park, Department of Dermatology, Soonchunhyang University Bucheon Hospital, 170 Jomaru-ro, Wonmi-gu, Bucheon 14584, Korea. Tel: 82-32-621-5062, Fax: 82-32-327-3550, E-mail: ylpark@schmc.ac.kr

ORCID: https://orcid.org/0000-0002-6532-3156

Si Hyung Lee, Department of Ophthalmology, Soonchunhyang University Bucheon Hospital, 170 Jomaru-ro, Wonmi-gu, Bucheon 14584, Korea. Tel: 82-32-621-5053, Fax: 82-32-327-3550, E-mail: sieh12@schmc.ac.kr ORCID: https://orcid.org/0000-0002-6383-4673

This is an Open Access article distributed under the terms of the Creative Commons Attribution Non-Commercial License (http://creativecommons. org/licenses/by-nc/4.0) which permits unrestricted non-commercial use, distribution, and reproduction in any medium, provided the original work is properly cited.

Copyright $($ c The Korean Dermatological Association and The Korean Society for Investigative Dermatology with AD had a higher prevalence of ophthalmic surgery compared to those without AD. Conclusion: Dermatologists should therefore be aware of possible ocular disorders in patients with $\mathrm{AD}$ and should recommend regular ophthalmic screening for early detection. (Ann Dermatol 31(1) $37 \sim 43$, 2019)

-Keywords-

Atopic dermatitis, Cataract, Glaucoma, Dry eye disease

\section{INTRODUCTION}

Atopic dermatitis (AD) is an inflammatory skin disorder characterized by relapsing eczema with intractable itching ${ }^{1}$. Besides well-known allergic conditions, including asthma, allergic rhinitis, and allergic conjunctivitis, many other comorbidities have recently been associated with $\mathrm{AD}^{2}$. Ocular disorders in patients with $\mathrm{AD}$ have been widely acknowledged and frequently reported worldwide. Specifically, cataract, retinal detachment, blepharitis, glaucoma, keratoconjunctivitis, and keratoconus are associated with $\mathrm{AD}^{3-6}$. However, the ocular disorders of $\mathrm{AD}$ patients are not of primary concern to most dermatologists, particularly if patients do not complain of associated symptoms. If left untreated, cataract and glaucoma in AD patients may progress to the advanced stages, resulting in irreversible vision loss.

To the best of our knowledge, only a few studies have used a large-sample, population-based study design to investigate possible relationships between $A D$ and ocular disease. The aim of the present study was therefore to use a population-based design to determine whether cataract, glaucoma, and dry eye disease are associated with $A D$ in 
an adult Korean population.

\section{MATERIALS AND METHODS}

\section{Study population}

This study was based on data from the 2010 2012 Korean National Health and Nutrition Examination Survey (KNHANES), a cross-sectional, population-based health examination and survey conducted by the Korea Centers for Disease Control and Prevention with Institutional Review Board approval ${ }^{7}$. The survey used multistage, stratified, probability cluster sampling to produce estimated health statistics representative of the noninstitutionalized civilian population of the Republic of Korea. The survey consisted of four components: a health-related interview, a health behavior survey, a health examination, and a nutrition survey. The interview included questions on demographics, socioeconomics, health, and nutrition. The health examination included vital signs, physiological measurements, and basic laboratory tests. We included subjects as AD patients who answered "yes" to the following question: "Have you ever been diagnosed as atopic dermatitis by doctor?." This study adhered to the tenets of the Declaration of Helsinki for biomedical research involving human subjects. Approval for this study was obtained from the institutional review board/Ethics Committee of Soonchunhyang University Bucheon Hospital (approval number SCHBC 2017-12-014).

\section{Ophthalmic examinations and surveys}

Participants underwent ophthalmic interviews, visual acuity measurements, intraocular pressure (IOP) measurements, autorefraction, slit-lamp examination, and fundus photography for their ophthalmic examinations. A past history of ophthalmic surgery and a family history of any ophthalmic disease were obtained from the interviews. The presence of dry eye disease was determined by following question: "Have you ever been diagnosed with dry eye disease by a doctor?" Available responses were "yes" or "no". If a participant answered "yes," he or she was classified as having clinical diagnosis of dry eye disease. A slit-lamp examination was performed to detect anterior segment abnormalities, evaluate the status of cataracts, and measure anterior chamber depth using the Van Herick method. The standard Lens Opacities Classification System (LOCS) III was used to categorize the type of cataract. Through a comparison with standard photographs, cataracts were categorized as cortical (LOCS III score $\geq 2$ ), nuclear (LOCS III score $\geq 4$ for nuclear opalescence or nuclear color), posterior subcapsular (PSC;
LOCS III score $\geq 2$ ), or mixed (more than one type of cataract per eye). The spherical equivalent was calculated as the sphere $+1 / 2$ cylinder. The IOP was measured once per eye from right to left with a Goldmann applanation tonometer (BQ-900; Haag-Streit, Bern, Switzerland) by a trained ophthalmologist. A digital non-mydriatic retina camera (TRC-NW6S; Topcon, Tokyo, Japan) and a Nikon D-80 digital camera (Nikon, Tokyo, Japan) were used to obtain digital fundus images under physiological mydriasis. Horizontal and vertical cup-to-disc ratios were measured from the fundus images, and features of diabetic retinopathy or age-related macular degeneration were checked.

A visual field test with frequency double technology was performed on participants with suspected glaucomatous features of the optic disc or an elevated IOP $(\geq 22$ $\mathrm{mmHg}$ ). Suspected glaucomatous features of the optic disc included (1) a horizontal or vertical cup to disc ratio (VCDR) $\geq 0.5$, (2) presence of optic disc hemorrhage, (3) presence of retinal nerve fiber layer defects, or (4) violation of the ISNT rule (i.e., normal eyes had a neuroretinal rim thickness configuration as follows: inferior $>$ superior $>$ nasal $>$ temporal). Open-angle glaucoma was diagnosed using modified International Society of Geographical and Epidemiological Ophthalmology criteria for the Korean population. The specific diagnostic criteria were as follows: category 1 required both (1) a reliable visual field defect consistent with glaucoma (a fixation error and false-positive error $\leq 1$ and the presence of at least two locations of reduced sensitivity) and (2) glaucomatous optic disc (neuroretinal rim loss with a VCDR or horizontal cup-to-disc ratio $\geq 0.6$, presence of optic disc hemorrhage, presence of retinal nerve fiber layer defects, or asymmetry of the VCDR $\geq 0.2$ ); category 2 , if a visual field test was not available or was unreliable (a fixation error or false-positive error $\geq 2$ ), required a VCDR $\geq 0.9$, asymmetry of the VCDR $\geq 0.3$, or presence of retinal nerve fiber layer defects and violation of the ISNT rule; category 3 required visual acuity $\leq 3 / 60$ and IOP $>21$ $\mathrm{mmHg}$. Subjects with a shallow anterior chamber (a peripheral anterior chamber depth less than or equal to one fourth of the central corneal thickness, indicating angle-closure glaucoma) were excluded.

\section{Other covariates}

Demographic and socioeconomic variables, including age, sex, urban or rural residence, income, and education level, were obtained from a health interview. Age was classified into 10-year intervals, income was stratified into quartiles, and education was categorized as elementary school or lower, middle school graduate, high school 
graduate and college graduate or higher. Regarding the region of residence, the 16 districts of the Republic of Korea were divided into two groups: (1) urban regions: Seoul, Gyeonggi, Busan, Daegu, Incheon, Gwangju, Daejeoun, and Ulsan; and (2) rural regions: Gangwon, Chungbuk, Chungnam, Jeonnam, Jeonbuk, Gyeongbuk, Gyeongnam, and Jeju. Occupation was classified into three groups: (1) white collar, comprising managers, professionals, clerical support workers, and service and sales workers; (2) blue collar, including agriculture, forestry, fishery, craft, and related trade workers; plant and machine operators and assemblers; and simple laborers; and (3) no occupation, including unemployed people, retired people, students, and homemakers. Body mass index (BMI) was calculated as follows: weight $(\mathrm{kg}) /$ height $\left(\mathrm{m}^{2}\right)$. Smoking status was categorized as smoker, ex-smoker, or nonsmoker. Drinking habits were classified according to drinking frequency as following: none, ocassional (less than 2 times per week), and frequent (more than 2 times per week).

Systolic and diastolic blood pressure (BP) were measured three times using a standard mercury sphygmomanometer (Baumanometer; WA Baum, Copiague, NY, USA) in a seated position, and the mean values for systolic and diastolic BP were recorded. Subjects were defined as having hypertension if they had a history of taking antihypertensive medications or had a BP $\geq 140 / 90 \mathrm{mmHg}$. Participants who had been diagnosed with diabetes by physicians or had a fasting glucose level $\geq 126 \mathrm{mg} / \mathrm{dl}$ were defined as having diabetes mellitus, and hypercholesterolemia was defined as having a fasting total cholesterol level $\geq 240 \mathrm{mg} / \mathrm{dl}$ or taking oral lipid-lowering agents.

\section{Statistical analysis}

Statistical analyses were performed using SAS for Windows, version 9.3 (SAS Institute, Cary, NC, USA). The PROC SURVEY procedure with sample weights was used to account for a complex sampling design and to produce nationally representative prevalence estimates. We presented the demographic characteristics of the participants according to the presence of glaucoma as either means \pm standard errors (SEs) or proportions and compared them between groups using the Rao-Scott chi-square test. We performed 4-to-1 matching by adjusting age and sex. Multiple logistic regression analyses were conducted to determine the associations between $\mathrm{AD}$ and various ocular disorders. An adjusted model was developed that included occupation and region as potential confounders. Odds ratios (ORs) and 95\% confidence intervals $(\mathrm{Cls})$ were also calculated. In all analyses, a two-tailed $p<0.05$ was considered statistically significant.

\section{RESULTS}

Of 25,534 participants in the 2010 2012 KNHANES, 10,634 were excluded for the following reasons: $<19$ years of age $(n=5,935)$, missing values for sample weights $(\mathrm{n}=3,212)$, and no responders for questions for $A D$ $(n=531)$, dry eye disease $(n=712)$, cataract $(n=53)$, or glaucoma $(n=191)$. Ultimately 14,900 participants were included in the statistical analyses: 14,598 non-AD subjects and 302 subjects with AD.

Demographic characteristics of the participants are shown in Table 1. Participants with AD were significantly younger $(p<0.001)$; were more highly educated $(p<0.001)$; were less frequent drinkers $(p=0.033)$; and had a lower prevalence of hypertension $(p<0.001)$, diabetes mellitus $(p<0.001)$, and hypercholesterolemia $(p=0.011)$ compared to the non-AD group. Significant difference in occupation was observed between non-AD and AD groups $(p<$ 0.001). After we performed 4-to-1 matching by adjusting age and sex (Table 2), the difference in occupation remained significant between non-AD and AD groups $(p=0.006)$ and AD participants were more likely to live in urban areas $(p<0.001)$. The prevalence and adjusted odd ratios for ocular diseases including dry eye disease, glaucoma, and cataract, and history of ocular surgery in matched participants are shown in Table 3. There were no significant differences between non-AD and $A D$ groups in the prevalence of ocular disease or history of ophthalmic surgery. Multiple logistic regression analyses were performed to identify associations between $\mathrm{AD}$ and ocular disease. After we adjusted for potential confounding factors, a history of ophthalmic surgery $(\mathrm{OR}, 2.90 ; 95 \% \mathrm{Cl}$, $1.33 \sim 6.30 ; p=0.007$ ), a history of cataract surgery (OR, $2.22 ; 95 \% \mathrm{Cl}, 1.07 \sim 4.61 ; p=0.032)$, a diagnosis of glaucoma (OR, $1.92 ; 95 \% \mathrm{Cl}, 1.11 \sim 3.32 ; p=0.020)$, and a diagnosis of cataract (OR, 1.85; 95\% Cl, 1.06 3.22; $p=0.031$ ) were significantly associated with AD. Of the five subtypes of cataracts, the cortical type (OR, 4.21; $95 \% \mathrm{Cl}, 1.58 \sim 10.86 ; p=0.004)$ and posterior subcapsular type (OR, 6.97; 95\% Cl, $2.66 \sim 19.48 ; p<0.001$ ) were significantly associated with AD.

\section{DISCUSSION}

Various ocular diseases are associated with $A D$, including cataract, retinal detachment, blepharitis, glaucoma, keratoconjunctivitis, and keratoconus ${ }^{4}$. This study characterized the possible association between AD and cataract, glaucoma, and dry eye disease in the adult population of the Republic of Korea using data obtained from the KNHANES. The prevalences of dry eye disease, glaucoma, 
Table 1. Baseline characteristics according to atopic dermatitis in Korean adults

\begin{tabular}{|c|c|c|c|}
\hline Variable & $\begin{array}{c}\text { Normal } \\
(n=14,598)\end{array}$ & $\begin{array}{c}A D \\
(n=302)\end{array}$ & $p$-value \\
\hline Age (yr) & & & $<0.001^{*}$ \\
\hline $19 \sim 29$ & $1,433(17.81)$ & 116 & \\
\hline $30 \sim 39$ & $2,508(20.27)$ & $56(15.8)$ & \\
\hline $40 \sim 49$ & $2,551(22.26)$ & $32(10.6)$ & \\
\hline $50 \sim 59$ & $2,878(18.91)$ & $41(10.83)$ & \\
\hline $60 \sim 69$ & $2,683(11.09)$ & $34(4.41)$ & \\
\hline$\geq 70$ & $2,545(9.65)$ & $23(2.52)$ & \\
\hline Sex & & & 0.428 \\
\hline Male & $5,842(49.04)$ & $114(51.85)$ & \\
\hline Female & $8,756(50.96)$ & 188 & \\
\hline House income & & & 0.597 \\
\hline Quartile 1 & $2,020(13.51)$ & $28(10.81)$ & \\
\hline Quartile 2 & $3,062(27.05)$ & $66(26.71)$ & \\
\hline Quartile 3 & $3,421(30.53)$ & $85(32.47)$ & \\
\hline Quartile 4 & $3,443(28.90)$ & $86(30.02)$ & \\
\hline Education level & & & $<0.001^{*}$ \\
\hline$\leq$ Elementary school & 2,695 (15.06) & $25(5.10)$ & \\
\hline Middle school graduate & e 1,269 (9.32) & $15(4.95)$ & \\
\hline High school graduate & $4,176(40.76)$ & $123(54.78)$ & \\
\hline$\geq$ College graduate & $3,910(34.86)$ & 108 & \\
\hline Occupation & & & $<0.001 *$ \\
\hline White collar & 4,479 (36.9) & $119(43.26)$ & \\
\hline Blue collar & $3,863(27.29)$ & $45(13.2)$ & \\
\hline Unemployed & $6,205(35.81)$ & $137(43.54)$ & \\
\hline Region & & & 0.068 \\
\hline Rural & $4,908(29.8)$ & $75(23.79)$ & \\
\hline Urban & $9,690(70.2)$ & $227(76.21)$ & \\
\hline Smoking status & & & 0.714 \\
\hline Nonsmoker & $8,923(54.37)$ & $185(57.34)$ & \\
\hline Ex-smoker & $2,998(20.72)$ & $60(19.09)$ & \\
\hline Smoker & $2,644(24.91)$ & $57(23.58)$ & \\
\hline Alcohol-drinking & & & $0.033^{*}$ \\
\hline None & $4,390(23.71)$ & $72(18.01)$ & \\
\hline Occasional ( $<2 / w k)$ & $7,289(53.74)$ & $179(62.78)$ & \\
\hline Frequent ( $\geq 2 / w k$ ) & $2,833(22.56)$ & $48(19.21)$ & \\
\hline $\begin{array}{l}\text { Overweight } \\
\left(\text { BMI } \geq 25 \mathrm{~kg} / \mathrm{m}^{2}\right)\end{array}$ & $4,612(32.05)$ & $84(29.46)$ & 0.457 \\
\hline Diagnosis of HTN & $1,469(24.26)$ & $16(10.18)$ & $<0.001^{*}$ \\
\hline Diagnosis of DM & $1,451(8.49)$ & $19(4.05)$ & $<0.001^{*}$ \\
\hline $\begin{array}{l}\text { Diagnosis of } \\
\text { hypercholesterolemia }\end{array}$ & $1,227(7.26)$ & $20(3.61)$ & $0.011 *$ \\
\hline $\begin{array}{l}\text { Family history of ocular } \\
\text { disease }\end{array}$ & $2,920(20.87)$ & $70(20.75)$ & 0.965 \\
\hline
\end{tabular}

Data are presented as numbers (weighted percentage). Numbers may not sum to 14,900 because of missing values in the database. AD: atopic dermatitis, BMI: body mass index, HTN: hypertension, DM: diabetes mellitus. p-values were calculated using the Rao-Scott chi-square test, and those in asterisks $\left({ }^{*}\right)$ are statistically significant.
Table 2. Baseline characteristics according to atopic dermatitis in Korean adults after matching for age and sex

\begin{tabular}{|c|c|c|c|}
\hline Variable & $\begin{array}{l}\text { Normal } \\
(n=1,208)\end{array}$ & $\begin{array}{c}\mathrm{AD} \\
(\mathrm{n}=302)\end{array}$ & $p$-value \\
\hline Age $(y r)$ & & & 0.993 \\
\hline $19 \sim 29$ & $461(54.52)$ & 116 & \\
\hline $30 \sim 39$ & $227(16.63)$ & $56(15.8)$ & \\
\hline $40 \sim 49$ & $129(10.83)$ & $32(10.6)$ & \\
\hline $50 \sim 59$ & $165(10.14)$ & $41(10.83)$ & \\
\hline $60 \sim 69$ & $128(4.71)$ & $34(4.41)$ & \\
\hline$\geq 70$ & $98(3.17)$ & $23(2.52)$ & \\
\hline Sex & & & 0.522 \\
\hline Male & 448 (49.35) & $114(51.85)$ & \\
\hline Female & $760(50.65)$ & $188(48.15)$ & \\
\hline House income & & & 0.759 \\
\hline Quartile 1 & $138(11.52)$ & $32(10.81)$ & \\
\hline Quartile 2 & $324(27.13)$ & $78(26.71)$ & \\
\hline Quartile 3 & $383(31.85)$ & $97(32.47)$ & \\
\hline Quartile 4 & $353(29.32)$ & $89(30.02)$ & \\
\hline Education level & & & 0.529 \\
\hline$\leq$ Elementary school & $121(6.34)$ & $24(5.10)$ & \\
\hline Middle school graduate & $117(6.12)$ & $23(4.95)$ & \\
\hline High school graduate & $572(52.53)$ & $152(54.78)$ & \\
\hline$\geq$ College graduate & $362(33.25)$ & $98(35.18)$ & \\
\hline Occupation & & & $0.006^{*}$ \\
\hline White collar & $451(40.15)$ & $119(43.26)$ & \\
\hline Blue collar & 301 (23.76) & $45(13.2)$ & \\
\hline Unemployed & $455(36.08)$ & $137(43.54)$ & \\
\hline Region & & & $<0.001^{*}$ \\
\hline Rural & $819(54.42)$ & $75(23.79)$ & \\
\hline Urban & $389(45.58)$ & $227(76.21)$ & \\
\hline Smoking status & & & 0.167 \\
\hline Nonsmoker & $805(60.1)$ & $185(57.34)$ & \\
\hline Ex-smoker & $171(13.61)$ & $60(19.09)$ & \\
\hline Smoker & $229(26.29)$ & $57(23.58)$ & \\
\hline Alcohol-drinking & & & 0.908 \\
\hline None & 285 (16.66) & $72(18.01)$ & \\
\hline Occasional $(<2 /$ wk $)$ & $720(63.73)$ & $179(62.78)$ & \\
\hline Frequent ( $\geq 2 / w k)$ & 199 (19.6) & $48(19.21)$ & \\
\hline $\begin{array}{l}\text { Overweight } \\
\qquad\left(\mathrm{BMl} \geq 25 \mathrm{~kg} / \mathrm{m}^{2}\right)\end{array}$ & 354 (28.94) & $84(29.46)$ & 0.891 \\
\hline Diagnosis of HTN & $170(8.89)$ & $42(6.35)$ & 0.143 \\
\hline Diagnosis of DM & $61(3.09)$ & $15(2.21)$ & 0.344 \\
\hline $\begin{array}{l}\text { Diagnosis of } \\
\text { hypercholesterolemia }\end{array}$ & $75(3.53)$ & $29(5.12)$ & 0.247 \\
\hline $\begin{array}{l}\text { Family history of ocular } \\
\text { disease }\end{array}$ & $281(22.04)$ & $70(20.75)$ & 0.674 \\
\hline
\end{tabular}

Data are presented as numbers (weighted percentage). Numbers may not sum to 1,510 because of missing values in the database. AD: atopic dermatitis, BMI: body mass index, HTN: hypertension, DM: diabetes mellitus. $p$-values were calculated using the Rao-Scott chi-square test, and those in asterisks $\left(^{*}\right)$ are statistically significant. 
Table 3. Prevalence and adjusted odds ratios for ocular diseases according to atopic dermatitis in Korean adults after matching for age and sex

\begin{tabular}{|c|c|c|c|c|c|}
\hline Variable & $\begin{array}{l}\text { Normal* } \\
(\mathrm{n}=1,208)\end{array}$ & $\begin{array}{c}A^{*} \\
(n=302)\end{array}$ & $p$-value ${ }^{\dagger}$ & $\begin{array}{c}\text { Adjusted OR } \\
(95 \% \quad \mathrm{Cl})^{\ddagger}\end{array}$ & $p$-value $e^{\S}$ \\
\hline $\begin{array}{l}\text { History of ophthalmic surgery } \\
\text { (glaucoma, cataract, retinal) }\end{array}$ & $1.82(0.38)$ & $2.68(0.81)$ & 0.288 & $2.90(1.33,6.30)$ & $0.007^{\|}$ \\
\hline History of glaucoma surgery & $0(0)$ & $0.53(0.53)$ & - & - & - \\
\hline History of cataract surgery & $1.82(0.38)$ & $2.15(0.61)$ & 0.635 & $2.22(1.07,4.61)$ & $0.032^{\|}$ \\
\hline Diagnosis of dry eye disease & $8.67(0.89)$ & $8.07(1.58)$ & 0.748 & $0.88(0.55,1.42)$ & 0.607 \\
\hline Diagnosis of glaucoma & $7.17(1.11)$ & $7.17(1.53)$ & 0.999 & $1.92(1.07,3.61)$ & $0.020^{\|}$ \\
\hline Diagnosis of cataract & $13.49(2.35)$ & $11.59(1.88)$ & 0.526 & $1.85(1.06,3.22)$ & $0.031^{\|}$ \\
\hline Type of cataract & & & 0.073 & & \\
\hline Cortical & $1.51(0.37)$ & $3.45(1.21)$ & & $4.21(1.58,10.86)$ & $0.004^{\|}$ \\
\hline Nuclear & $8.54(1.96)$ & $6.01(1.36)$ & & $1.39(0.72,2.68)$ & 0.328 \\
\hline Anterior (sub) capsular & $0.18(0.13)$ & $0.05(0.05)$ & & - & - \\
\hline Posterior (sub) capsular & $0.02(0.02)$ & $0.03(0.03)$ & & $6.97(2.66,19.48)$ & $<0.001^{\|}$ \\
\hline Mixed & $1.76(0.56)$ & $0.80(0.43)$ & & $1.36(0.46,4.05)$ & 0.583 \\
\hline
\end{tabular}

AD: atopic dermatitis, OR: odds ratio, $\mathrm{Cl}$ : confidence interval. *Percentages (standard errors) are calculated based on weighted data to better represent the study population. ${ }^{\dagger} p$-values were calculated using the Rao-Scott chi-square test. ${ }^{\dagger}$ Adjusted for occupation and region. ${ }^{\S} p$-values for adjusted odds ratios. "Significant $p$-values.

and cataract in adults with $\mathrm{AD}$ were estimated to be $8.07 \%, 7.17 \%$, and $11.59 \%$, respectively in this study. After we adjusted for confounding factors, we found that patients with AD had a higher prevalence of ophthalmic surgery, in particular cataract surgery, compared to the normal control group. Patients with AD had a greater likelihood of having been diagnosed with glaucoma and cataract compared to non-AD subjects. Of the five subtypes of cataracts, cortical and posterior subcapsular cataracts were significantly associated with AD. However, an association between dry eye disease and AD was not found.

Cataract is one of the most well-known ocular complications of AD. Because there are not many well-organized studies, the exact prevalence of cataract in patients with $\mathrm{AD}$ is currently unknown, but it varies from $1 \%$ to $38 \% 6,8,9$. In our study, it was estimated to be $11.59 \%$, which is consistent with previous studies. The pathogenesis of cataract in AD is not definitively known. There may be multiple causes, including direct trauma, inflammatory processes associated with $A D$, and long-term use of corticosteroids ${ }^{9-12}$. Oxidative stress in AD patients has also frequently been suggested as a main contributing factor to developing cataracts ${ }^{9}$. A higher level of serum lipid peroxide produced from unsaturated fatty acids and a lower level of superoxide dismutase were observed in AD patients with cataracts compared to AD patients without cataracts $^{9,13}$, irrespective of previous topical corticosteroid treatments ${ }^{13}$. Decreased activity by superoxide dismutase, which scavenges reactive oxygen species (ROS) and inhibits the formation of free radicals, has been reported in
AD patients with cataracts $^{9,13}$. Elevated serum IgE levels and genetic factors associated with AD may also be associated with the formation of cataracts ${ }^{14}$.

Among the many subtypes of cataracts, subcapsular cataracts have typically been reported as being associated with AD. Although the anterior subscapular cataract (ASC) is more specifically related to $A D$, the PSC is more common in AD patients ${ }^{3,12,15}$. In our study, AD was associated with PSCs, consistent with previous studies. However, an association with ASCs could not be determined because the prevalence of ASC was very low. The reason for the low prevalence of both ASC and PSC in this study is probably the large number of patients undergoing early cataract surgery for these two cataract subtypes, because the location of lens opacification is relatively central to the visual axis and thus may impair visual acuity more severely. The significant association between AD and cataract surgery found in this study is consistent with this hypothesis.

The exact pathogenesis of the ASC is still not known. However, the ASC is neither an age-related nor steroid-induced cataract ${ }^{16}$. It is probably closely related to AD because $A D$ has been suggested as the most common cause of the $\mathrm{ASC}^{3,12,15}$. The ASC tends to develop in early child$\operatorname{hood}^{16}$, and repeated physical trauma due to rubbing and scratching the eyes plays an important role ${ }^{9}$. Steroid treatment is the most well-known factor contributing to the formation of the PSC $3,6,9,17,18$. This treatment disturbs the normal metabolism of ocular cells, resulting in osmotic failure, thus making lens proteins more vulnerable to oxida- 
tive stress ${ }^{18}$. Because AD is a chronic inflammatory skin disorder that often requires long-term corticosteroid use, the association between PSC and AD seems reasonable. However, another study reported no differences in the incidence of cataracts between corticosteroid-naive AD patients and AD patients with a history of corticosteroid use, which suggests that the PSC in patients with AD cannot be fully explained by the use of corticosteroids alone ${ }^{13}$.

Our study showed that cortical cataracts were also associated with AD. Known risk factors for cortical cataracts include age, female sex, genetic factors, diabetes mellitus, and ultraviolet $B$ light (UVB) ${ }^{10}$. In our study, the association between $\mathrm{AD}$ and the cortical cataract subtype was present even after we adjusted for age, sex, and diabetes mellitus, which suggests that other factors may have influenced the formation of cortical cataracts. Among these risk factors, the relationship between UVB and cataract has frequently been suggested ${ }^{10,19,20}$. Patients with $A D$ may have a greater chance of being exposed to narrowband UVB, because narrowband UVB is widely used in chronic AD treatment. Having unprotected eyes during therapy may increase the risk of UVB exposure among AD patients ${ }^{9}$.

The relationship between $\mathrm{AD}$ and glaucoma has often been reported since Harris ${ }^{21}$ first suggested glaucoma as an adverse effect of glucocorticoid treatment in 1960. Although a recent study from Denmark reported no relationship between glaucoma and $\mathrm{AD}^{4}$, many studies have reported a positive association between glaucoma and $A D$, which is consistent with the results of our study. Steroid administration has frequently been suggested as the single most important cause of the development of glaucoma in patients with AD. The elevated IOP induced by the use of corticosteroids may cause optic nerve damage, resulting in glaucoma ${ }^{3,22}$. Recently, an increased inflammatory response in AD was suggested as another possible factor contributing to the development of glaucoma in AD patients. The study reported that inflammatory cytokines such as IL-8 and CCL2 were increased in glaucoma patients with $\mathrm{AD}^{22}$.

Dry eye disease has been suggested as a related symptom of atopic keratoconjunctivitis (AKC), which is a severe ocular complication in AD patients ${ }^{5,23}$. Abnormal tear functioning, including meibomian gland dysfunction and decreased tear film break-up time, have been suggested as the main pathogenic mechanisms involved in developing irritation and dry eye in patients with $\mathrm{AKC}^{5,23}$. Goblet cell loss and conjunctival squamous metaplasia may also contribute to lacrimal film instability in patients with $A K C^{5}$. However, whether dry eye syndrome can develop independently from AKC in patients with AD has not been definitively established, and further studies are needed to confirm this relationship. The results of the present study showed no significant association between AD and a diagnosis of dry eye disease by clinicians. One possible reason for this is that the diagnosis of dry eye disease was based on self-report questionnaires; most of the participants with AD may have had a doctor's diagnosis of AKC but not a specific diagnosis of dry eye disease.

The present study has some limitations. First, given the cross-sectional nature of the study, causal relationships between $\mathrm{AD}$ and the ocular diseases cannot be definitively established. Second, because the KNHANES is based on self-reported data, there was the potential for incorrect classifications by the participants. Third, although we included several potential confounders, unidentified factors may have contributed to these associations. Fourth, the route of administration, dose, frequency, and duration of corticosteroid treatments are important factors in the pathogenesis of cataract and glaucoma in AD patients ${ }^{24-26}$, but these data were not provided by KNHANES. Nonetheless, a strength of the present study is that it used data from a nationwide survey that included a large population, which minimizes selection bias. In addition, because few population-based studies have investigated possible associations between ocular disease and AD worldwide, our study may help to confirm and expand previous findings of associations between cataract and glaucoma in patients with AD.

In summary, this is the first study to comprehensively investigate the associations between $A D$ and selected ocular disorders in the Republic of Korea. The results showed that cataract and glaucoma were significantly associated with $\mathrm{AD}$ in adult patients. Furthermore, there was a significantly higher prevalence of ophthalmic surgery among AD patients than non-AD patients, which implies that ocular diseases occurring in patients with $\mathrm{AD}$ require more frequent surgical interventions. Dermatologists should therefore be aware of ocular manifestations in patients with $\mathrm{AD}$ and should recommend regular screening of these patients for the early detection of ocular disorders.

\section{ACKNOWLEDGMENT}

This work was supported by the National Research Foundation of Korea (NRF) grant funded by the Korea Government (Ministry of Education) (No. 2017R1D1A1B0 3029944) and by the Soonchunhyang University Research Fund.

\section{CONFLICTS OF INTEREST}

The authors have nothing to disclose. 


\section{ORCID}

Sul Hee Lee, https://orcid.org/0000-0002-2990-9774

Sang-Hoon Lee, https://orcid.org/0000-0002-7146-3702

Si Hyung Lee, https://orcid.org/0000-0002-6383-4673

Young Lip Park, https://orcid.org/0000-0002-6532-3156

\section{REFERENCES}

1. Weidinger S, Novak N. Atopic dermatitis. Lancet 2016;387: 1109-1122.

2. Brunner PM, Silverberg JI, Guttman-Yassky E, Paller AS, Kabashima $K$, Amagai $M$, et al. Increasing comorbidities suggest that atopic dermatitis is a systemic disorder. J Invest Dermatol 2017; 137:18-25.

3. Bercovitch L. Screening for ocular complications in atopic dermatitis. Arch Dermatol 2011;147:588-589.

4. Thyssen JP, Toft PB, Halling-Overgaard AS, Gislason GH, Skov L, Egeberg A. Incidence, prevalence, and risk of selected ocular disease in adults with atopic dermatitis. J Am Acad Dermatol 2017;77:280-286.e1.

5. Chen JJ, Applebaum DS, Sun GS, Pflugfelder SC. Atopic keratoconjunctivitis: a review. J Am Acad Dermatol 2014; 70:569-575.

6. Haeck IM, Rouwen TJ, Timmer-de Mik L, de Bruin-Weller MS, Bruijnzeel-Koomen CA. Topical corticosteroids in atopic dermatitis and the risk of glaucoma and cataracts. J Am Acad Dermatol 2011;64:275-281.

7. Kweon S, Kim Y, Jang MJ, Kim Y, Kim K, Choi S, et al. Data resource profile: the Korea National Health and Nutrition Examination Survey (KNHANES). Int J Epidemiol 2014;43: 69-77.

8. Chen CC, Huang JL, Yang KD, Chen HJ. Atopic cataracts in a child with atopic dermatitis: a case report and review of the literature. Asian Pac J Allergy Immunol 2000;18:69-71.

9. Bair B, Dodd J, Heidelberg K, Krach K. Cataracts in atopic dermatitis: a case presentation and review of the literature. Arch Dermatol 2011;147:585-588.

10. Asbell PA, Dualan I, Mindel J, Brocks D, Ahmad M, Epstein S. Age-related cataract. Lancet 2005;365:599-609.

11. Skalka HW, Prchal JT. Effect of corticosteroids on cataract formation. Arch Ophthalmol 1980;98:1773-1777.

12. Castrow FF 2nd. Atopic cataracts versus steroid cataracts. J Am Acad Dermatol 1981;5:64-66.

13. Niwa $Y$, lizawa $O$. Abnormalities in serum lipids and leukocyte superoxide dismutase and associated cataract formation in patients with atopic dermatitis. Arch Dermatol 1994;130:1387-1392.

14. Bielory B, Bielory L. Atopic dermatitis and keratoconjunctivitis. Immunol Allergy Clin North Am 2010;30:323-336.

15. Amemiya $T$, Matsuda $H$, Uehara $M$. Ocular findings in atopic dermatitis with special reference to the clinical features of atopic cataract. Ophthalmologica 1980;180:129-132.

16. Na KS, Park YG, Han K, Mok JW, Joo CK. Prevalence of and risk factors for age-related and anterior polar cataracts in a Korean population. PLoS One 2014;9:e96461.

17. Daniel BS, Orchard D. Ocular side-effects of topical corticosteroids: what a dermatologist needs to know. Australas J Dermatol 2015;56:164-169.

18. Jobling AI, Augusteyn RC. What causes steroid cataracts? A review of steroid-induced posterior subcapsular cataracts. Clin Exp Optom 2002;85:61-75.

19. Hollows F, Moran D. Cataract--the ultraviolet risk factor. Lancet 1981;2:1249-1250.

20. Hightower KR. A review of the evidence that ultraviolet irradiation is a risk factor in cataractogenesis. Doc Ophthalmol 1994;88:205-220.

21. Harris JL. Glaucoma associated with steroid therapy and atopic dermatitis. Am J Ophthalmol 1960;49:351-353.

22. Takakuwa K, Hamanaka T, Mori K, Chin S, Shinmei Y, Funaki T, et al. Atopic glaucoma: clinical and pathophysiological analysis. J Glaucoma 2015;24:662-668.

23. Dogru M, Nakagawa N, Tetsumoto K, Katakami C, Yamamoto M. Ocular surface disease in atopic dermatitis. Jpn J Ophthalmol 1999;43:53-57.

24. Butcher JM, Austin M, McGalliard J, Bourke RD. Bilateral cataracts and glaucoma induced by long term use of steroid eye drops. BMJ 1994;309:43.

25. Garbe E, LeLorier J, Boivin JF, Suissa S. Risk of ocular hypertension or open-angle glaucoma in elderly patients on oral glucocorticoids. Lancet 1997;350:979-982.

26. Kersey JP, Broadway DC. Corticosteroid-induced glaucoma: a review of the literature. Eye (Lond) 2006;20:407-416. 